Proyecciones Journal of Mathematics

Vol. 34, $\mathrm{N}^{\circ}$ 4, pp. 307-322, December 2015.

Universidad Católica del Norte

Antofagasta - Chile

\title{
The multi-step homotopy analysis method for solving fractional-order model for HIV infection of $\mathrm{CD}^{+} \mathrm{T}$ cells
}

\author{
Ali H. Handam \\ Al Al-Bayt University, Jordan \\ Asad A. Freihat \\ Ajloun College Al-Balqa Applied University, Jordan \\ and \\ M. Zurigat \\ Al Al-Bayt University, Jordan \\ Received : March 2015. Accepted : October 2015
}

\begin{abstract}
$H I V$ infection of $\mathrm{CD}_{4}{ }^{+} \mathrm{T}$ cells is one of the causes of health problems and continues to be one of the significant health challenges. This paper presents approximate analytical solutions to the model of HIV infection of $\mathrm{CD}_{4}^{+} \mathrm{T}$ cells of fractional order using the multi-step homotopy analysis method (MHAM). The proposed scheme is only a simple modification of the homotopy analysis method (HAM), in which it is treated as an algorithm in a sequence of small intervals (i.e. time step) for finding accurate approximate solutions to the corresponding problems. The fractional derivatives are described in the Caputo sense. A comparative study between the new algorithm and the classical Runge-Kutta method is presented in the case of integer-order derivatives. The solutions obtained are also presented graphically.
\end{abstract}

Subjclass [2000] : 26A33, 34A34.

Keywords : Fractional-order, Caputo fractional derivative, multistep homotopy analysis, HIV infection, Differential equation. 


\section{Introduction}

$\mathrm{HIV}$ is a retrovirus that targets the $\mathrm{CD} 4^{+} \mathrm{T}$ lymphocytes, which are the most abundant white blood cells of the immune system. Although HIV infects other cells also, it wreaks the most havoc on the $\mathrm{CD} 4^{+} \mathrm{T}$ cells by causing their decline and destruction, thus decreasing the resistance of the immune system. A number of mathematical models have been proposed to understand HIV dynamics, disease progression, anti-retroviral response etc $[11,12,13,14]$.

In 1989, Perelson [12] developed a simple model for the primary infection with HIV. Perelson et al. [13] extended Perelson's model and proved mathematically some of the model's behavior. They observed that the model exhibits many of the symptoms of AIDS seen clinically: the long latency period, low levels of free virus in the body, and the depletion of $\mathrm{CD} 4^{+} \mathrm{T}$ cells. They defined the model by considering four compartments: cells that are uninfected, cells that are latently infected, cells that are actively infected and free virus. They described the dynamics of these populations by a system of four ordinary differential equations. This model has been important in the field of mathematical modelling of HIV infection, and many other models have been proposed, which take the model of Perelson, Kirschner and De Boer as their inspiration. In the paper [3], Culshaw and Ruan simplify the model of Perelson, Kirschner and De Boer into one consisting of only three components: the healthy $\mathrm{CD} 4^{+} \mathrm{T}$ cells, infected $\mathrm{CD} 4^{+} \mathrm{T}$ cells, and free virus, and introduce a discrete time delay to the model.

Nowadays several researchers work on the fractional order differential equations because of best presentation of many phenomena. Fractional calculus has been used to model physical and engineering processes, which are found to be best described by fractional differential equations. It is worth nothing that the standard mathematical models of integer-order derivatives, including nonlinear models, do not work adequately in many cases. In the recent years, fractional calculus has played a very important role in various fields such as mechanics, electricity, chemistry, biology, economics, notably control theory, and signal and image processing see for example $[4,5,7,9]$. In this paper, we investigate the applicability and effectiveness of the homotopy analysis method when treated as an algorithm in a sequence of intervals (i.e. time step) for finding accurate approximate solutions to model for HIV infection of $\mathrm{CD}^{+} \mathrm{T}$ cells. This modified method is named as the multi-step homotopy analysis method. It can be found that the corresponding numerical solutions obtained by using HAM are valid 
only for a short time. While the ones obtained by using MHAM are more valid and accurate during a long time [1]. In this paper, we intend to obtain the approximate solution of the fractional-order model for HIV infection of $\mathrm{CD}^{+} \mathrm{T}$ cells via the multi-step homotopy analysis method. Finally we compare our numerical results with nonstandard numerical method and fourth order Runge-Kutta method.

\section{Model description}

In this study we consider the model for HIV infection of $\mathrm{CD} 4^{+} \mathrm{T}$ cells taking by Merdan [8] and extend it to the new system of the following set of fractional order differential equations of order $\alpha>0$ :

$$
\begin{gathered}
D^{\alpha} \grave{T}(t)=s-\delta \grave{T}(t)+r \grave{T}(t)\left(1-\frac{\grave{T}(t)+I(t)}{\grave{T}_{m}}\right)-k V(t) \grave{T}(t), \\
D^{\alpha} I(t)=k V(t) \grave{T}(t)-\beta I(t), \\
D^{\alpha} V(t)=N \beta I(t)-\gamma V(t) .
\end{gathered}
$$

where $\grave{T}(t)$ represents the concentration of healthy $\mathrm{CD} 4^{+} \mathrm{T}$ cells at time $t$, $I(t)$ represents the concentration of infected $\mathrm{CD} 4^{+} \mathrm{T}$ cells, and $V(t)$ the concentration of free HIV at time $t$. Following, we note that $s$ is the source of $\mathrm{CD} 4^{+} \mathrm{T}$ cells from precursors, $\delta$ is the natural death rate of $\mathrm{CD} 4^{+} \mathrm{T}$ cells $\left(\delta \grave{T}_{m}>s\right), r$ is their growth rate, and $\grave{T}_{m}$ is their carrying capacity. The parameter $k$ represents the rate of infection of T cells with free virus. $\beta$ is a blanket death term for infected cells, to reflect the assumption that we do not initially know whether the cells die naturally or by bursting. Since $N$ viral particles are released by each lysing cell, this term is multiplied by the parameter $N$ to represent the source for free virus (assuming a onetime initial infection). Finally, $\gamma$ is the loss rate of virus. Throughout this paper, we set the values of the system parameters as $s=0.1, \delta=0.02, \beta$ $=0.3, r=3, \gamma=2.4, k=0.0027, N=10, \grave{T}_{m}=1500$, and initial densities $\grave{T}(0)=0.001, I(0)=0, V(0)=0.001$.

\section{Fractional calculus}

In this section, we give some basic definitions and properties of the fractional calculus theory which are used further in this paper. 
Definition 3.1. A function $f(x)(x>0)$ is said to be in the space $C_{\alpha}$ $(\alpha \in \mathbf{R})$ if it can be written as $f(x)=x^{p} f_{1}(x)$ for some $p>\alpha$ where $f_{1}(x)$ is continuous in $[0, \infty)$, and it is said to be in the space $C_{\alpha}^{m}$ if $f^{(m)} \in$ $C_{\alpha}, m \in \mathbf{N}$.

Definition 3.2. The Riemann-Liouville integral operator of order $\alpha$ with $a \geq 0$ is defined as

$$
\begin{gathered}
\left(J_{a}^{\alpha} f\right)(x)=\frac{1}{\Gamma(\alpha)} \int_{a}^{x}(x-t)^{\alpha-1} f(t) d t, \quad x>a, \\
\left(J_{a}^{0} f\right)(x)=f(x) .
\end{gathered}
$$

Properties of the operator can be found in $[6,9,10,15,17]$. For $f \in C_{\alpha}$, $\alpha, \beta>0, a \geq 0, c \in \mathbf{R}, \gamma>-1$, we have

$$
\begin{gathered}
\left(J_{a}^{\alpha} J_{a}^{\beta} f\right)(x)=\left(J_{a}^{\beta} J_{a}^{\alpha} f\right)(x)=\left(J_{a}^{\alpha+\beta} f\right)(x), \\
J_{a}^{\alpha} x^{\gamma}=\frac{x^{\gamma+\alpha}}{\Gamma(\alpha)} 0 B_{\frac{x-a}{x}}(\alpha, \gamma+1),
\end{gathered}
$$

where $B_{\tau}(\alpha, \gamma+1)$ is the incomplete beta function which is defined as

$$
\begin{gathered}
B_{\tau}(\alpha, \gamma+1)=\int_{0}^{\tau} t^{\alpha-1}(1-t)^{\gamma} d t \\
J_{a}^{\alpha} e^{c x}=e^{a c}(x-a)^{\alpha} \sum_{k=0}^{\infty} \frac{[c(x-a)]^{k}}{\Gamma(\alpha+k+1)} .
\end{gathered}
$$

The Riemann-Liouville derivative has certain disadvantages when trying to model real-world phenomena with fractional differential equations. Therefore, we shall introduce a modified fractional differential operator $D_{a}^{\alpha}$ proposed by Caputo in his work on the theory of viscoelasticity.

Definition 3.3. The Caputo fractional derivative of $f(x)$ of order $\alpha>0$ with $a \geq 0$ is defined as

$$
\left(D_{a}^{\alpha} f\right)(x)=\left(J_{a}^{m-\alpha} f^{(m)}\right)(x)=\frac{1}{\Gamma(m-\alpha)} \int_{a}^{x} \frac{f^{(m)}(t)}{(x-t)^{\alpha+1-m}} d t
$$


for $m-1<\alpha \leq m, m \in \mathbf{N}, x \geq a, f \in C_{-1}^{m}$.

The Caputo fractional derivative was investigated by many authors, for $m-1<\alpha \leq m, f(x) \in C_{\alpha}^{m}$, we have

$$
\left(J_{a}^{\alpha} D_{a}^{\alpha} f\right)(x)=J^{m} D^{m} f(x)=f(x)-\sum_{k=0}^{m-1} f^{(k)}(a) \frac{(x-a)^{k}}{k !} .
$$

For mathematical properties of fractional derivatives and integrals one can consult the mentioned references.

\section{Multi-step homotopy analysis method}

The MHAM is used to provide approximate solutions for nonlinear problem in terms of convergent series with easily computable components, it has been shown that the approximated solution obtained are not valid for large $\mathrm{t}$ for some systems. Therefore we use the MHAM, which is offers accurate solution over a longer time frame compared to the HAM [1, 2, 16, 18, 19]. In this section we need to construct the MHAM of the general form of the HIV infection of $\mathrm{CD} 4^{+} \mathrm{T}$ cells model (2.1). For this purpose, we consider the following fractional system of differential equations

$$
D^{\alpha} x_{i}(t)=F_{i}\left(t, x_{1}(t), \ldots, x_{r}(t)\right), \quad t \geq 0,0<\alpha \leq 1,
$$

subject to the initial conditions

$$
x_{i}(0)=d_{i}, \quad i=1,2, \ldots, r,
$$

where $\left(F_{i}\left(t, x_{1}(t), \ldots, x_{r}(t)\right), i=1,2, \ldots, r\right)$ are known analytical functions. Let $[0, T]$ be the interval over which we want to find the solution of the initial value problem (4.1) and (4.2). Assume that the interval $[0, T]$ is divided into $n$-subintervals of equal length $\Delta t,\left[t_{0}, t_{1}\right],\left[t_{1}, t_{2}\right],\left[t_{2}, t_{3}\right], \ldots,\left[t_{n-1}, t_{n}\right]$ with $t_{0}=0, t_{n}=T$. Let $t^{*}$ be the initial value for each subintervals and let $x_{i, j}(t), i=1,2, \ldots, r, j=1,2, \ldots, n$ be approximate solutions in each subinterval $\left[t_{j-1}, t_{j}\right], j=1,2, \ldots, n$, with initial guesses

$x_{i, 1}\left(t^{*}\right)=d_{i}, x_{i, j}\left(t^{*}\right)=X_{i, j}\left(t_{j-1}\right)=X_{i, j-1}\left(t_{j-1}\right), i=1,2, \ldots, r, j=2,3, \ldots, n$.

Now, we can construct the so-called zeroth-order deformation equations of the system (4.1) by 
$(1-q) L\left[\phi_{i, j}(t ; q)-x_{i, j}\left(t^{*}\right)\right]=q h\left[D_{t}^{\alpha} \phi_{i, j}(t ; q)-F_{i}\left(t, \phi_{1, j}(t ; q), \ldots, \phi_{r, j}(t ; q)\right)\right]$

$$
i=1,2, \ldots, r, \quad j=1,2, \ldots, n,
$$

where $q \in[0,1]$ is an embedding parameter, $L$ is an auxiliary linear operator, $h \neq 0$ is an auxiliary parameter and $\phi_{i, j}(t ; q)$ are unknown functions. Obviously, when $q=0$

$$
\phi_{i, 1}(t ; 0)=d_{i}, \quad \phi_{i, j}(t ; 0)=x_{i, j-1}\left(t_{j-1}\right), i=1,2, \ldots, r, \quad j=2,3, \ldots, n,
$$

and when $q=1$, we have

$$
\phi_{i, j}(t ; 1)=x_{i, j}(t), \quad i=1,2, \ldots, r, j=1,2, \ldots, n \text {. }
$$

Expanding $\phi_{i, j}(t ; q), i=1,2, \ldots, r, j=1,2, \ldots, n$, in Taylor series with respect to $q$, we get

$$
\phi_{i, j}(t ; q)=x_{i, j}\left(t^{*}\right)+\sum_{m=1}^{\infty} x_{i, j, m}(t) q^{m}, i=1,2, \ldots, r, j=1,2, \ldots, n,
$$

where

$$
x_{i, j, m}(t)=\left.\frac{1}{m !} \frac{\partial^{m} \phi_{i, j}(t ; q)}{\partial q^{m}}\right|_{q=0} .
$$

If the initial guesses $x_{i, j}\left(t^{*}\right)$, the auxiliary linear operator $L$ and the nonzero auxiliary parameter $h$ are properly chosen so that the power series (4.5) converges at $q=1$, one has

$$
x_{i, j}(t)=\phi_{i, j}(t ; 1)=x_{i, j}\left(t^{*}\right)+\sum_{m=1}^{\infty} x_{i, j, m}(t) .
$$

Define the vector

$$
\vec{x}_{i, j, m}(t)=\left\{x_{i, j, 0}(t), x_{i, j, 1}(t), \ldots, x_{i, j, m}(t)\right\} .
$$


Differentiating the zero-order deformation equation (4.4) $m$ times with respective to $q$, then setting $q=0$ and dividing them by $m$ !, finally using (4.6), we have the so-called high-order deformation equations

$$
L\left[x_{i, j, m}(t)-\chi_{m} x_{i, j, m-1}(t)\right]=h \Re_{i, j, m}\left(\vec{x}_{i, j, m-1}(t)\right),
$$

subject to the initial conditions

$$
x_{i, j, m}(0)=0, \quad i=1,2, \ldots, r, \quad j=1,2, \ldots, n,
$$

where

$$
\begin{gathered}
\Re_{i, j, m}\left(\vec{x}_{i, j, m-1}(t)\right) \\
=\left.\frac{1}{(m-1) !} \frac{\partial^{m-1}}{\partial q^{m-1}}\left[D_{t}^{\alpha} \phi_{i, j}(t ; q)-F_{i}\left(t, \phi_{1, j}(t ; q), \ldots, \phi_{r, j}(t ; q)\right)\right]\right|_{q=0}, \\
=1,2, \ldots, r, \quad j=1,2, \ldots, n,
\end{gathered}
$$

and

$$
\chi_{m}=\left\{\begin{array}{cc}
0, & m \leq 1 \\
1, & m>1
\end{array} .\right.
$$

Select the auxiliary linear operator $L=D^{\alpha}$, then the $\mathrm{m}^{\text {th }}$ order deformation equations (4.8) can be written in the form

$x_{i, j, m}(t)=\chi_{m} x_{i, j, m-1}(t)+h J^{\alpha}\left[\Re_{i, j, m}\left(\vec{x}_{i, j, m-1}(t)\right)\right], i=1,2, \ldots, r, \quad j=1,2, \ldots, n$.

The solutions of system (4.1) in each subinterval $\left[t_{j-1}, t_{j}\right], j=1,2, \ldots, n$, has the form

$$
X_{i, j}(t)=\sum_{m=0}^{\infty} x_{i, j, m}\left(t-t_{j-1}\right), i=1,2, \ldots, r, j=1,2, \ldots, n,
$$

and the solution of system (4.1) for $[0, T]$ is given by

$$
x_{i}(t)=\sum_{j=1}^{n} \chi_{v} X_{i, j}(t), i=1,2, \ldots, r,
$$

where $\chi_{v}= \begin{cases}1, & t \in\left[t_{j-1}, t_{j}\right] \\ 0, & t \notin\left[t_{j-1}, t_{j}\right]\end{cases}$ 
Theorem 4.1. If the series $\sum_{m=0}^{\infty} x_{i, j, m}(t), i=1,2, \ldots, r, j=1,2, \ldots, n$, is convergent, it must be a solution of system (4.1).

Proof. As we say the series $\sum_{m=0}^{\infty} x_{i, j, m}(t)$ is convergent, then $m \longrightarrow \infty \lim x_{i, j, m}(t)=0$, for all $i, j$. Using the notation of $\chi_{m}$, we have

$$
\sum_{m=1}^{k}\left[x_{i, j, m}(t)-\chi_{m} x_{i, j, m-1}(t)\right]=x_{i, j, k}(t)
$$

which gives us

$$
\sum_{m=1}^{\infty}\left[x_{i, j, m}(t)-\chi_{m} x_{i, j, m-1}(t)\right]=k \longrightarrow \infty \lim x_{i, j, k}(t)=0 .
$$

Furthermore, using the above expression and the equation (4.8), we have

$$
\sum_{m=1}^{\infty} L\left[x_{i, j, m}(t)-\chi_{m} x_{i, j, m-1}(t)\right]=h \sum_{m=1}^{\infty} \Re_{i, j, m}\left(\vec{x}_{i, j, m-1}(t)\right)=0
$$

which gives, since $h \neq 0$ that $\sum_{m=1}^{\infty} \Re_{i, j, m}\left(\vec{x}_{i, j, m-1}(t)\right)=0$, that is

$$
\begin{aligned}
& \left.\sum_{m=1}^{\infty} \frac{1}{(m-1) !} \frac{\partial^{m-1}}{\partial q^{m-1}}\left[D_{t}^{\alpha} \phi_{i, j}(t ; q)-F_{i}\left(t, \phi_{1, j}(t ; q), \ldots, \phi_{r, j}(t ; q)\right)\right]\right|_{q=0} \\
& =\left.\sum_{m=0}^{\infty} \frac{1}{m !} \frac{\partial^{m}}{\partial q^{m}}\left[D_{t}^{\alpha} \phi_{i, j}(t ; q)-F_{i}\left(t, \phi_{1, j}(t ; q), \ldots, \phi_{r, j}(t ; q)\right)\right]\right|_{q=0}=0 .
\end{aligned}
$$

In additional, $\phi_{i, j}(t ; q)$ are not a solutions of (4.1) on an interval $\left[t_{j-1}, t_{j}\right]$ when $q \neq 0$. Now define

$$
\begin{aligned}
\lambda_{i, j}(t, q)= & D_{t}^{\alpha} \phi_{i, j}(t ; q)-F_{i}\left(t, \phi_{1, j}(t ; q), \ldots, \phi_{r, j}(t ; q)\right) \\
& -\left(D_{t}^{\alpha} x_{i, j}(t)-F_{i}\left(t, x_{1, j}, \ldots, x_{m, j}\right)\right) \\
= & D_{t}^{\alpha} \phi_{i, j}(t ; q)-F_{i}\left(t, \phi_{1, j}(t ; q), \ldots, \phi_{r, j}(t ; q)\right)
\end{aligned}
$$


as a residual error of system (4.1), then the Maclaurin's series of $\lambda_{i, j}(t, q)$ with respect to empedding parameter $q$ is:

$$
\begin{aligned}
& \left.\sum_{m=0}^{\infty} \frac{q^{m}}{m !} \frac{\partial^{m}}{\partial q^{m}} \lambda_{i, j}(t, q)\right|_{q=0} \\
= & \left.\sum_{m=0}^{\infty} \frac{q^{m}}{m !} \frac{\partial^{m}}{\partial q^{m}}\left[D_{t}^{\alpha} \phi_{i, j}(t ; q)-F_{i}\left(t, \phi_{1, j}(t ; q), \ldots, \phi_{r, j}(t ; q)\right)\right]\right|_{q=0} .
\end{aligned}
$$

When $q=1$, and upon using equation (4.14), the above expression leads to

$$
\lambda_{i, j}(t, 1)=\left.\sum_{m=0}^{\infty} \frac{1}{m !} \frac{\partial^{m}}{\partial q^{m}} \lambda_{i, j}(t, q)\right|_{q=0}=0,
$$

which means that $\phi_{i, j}(t ; 1)$ must be a solutions of system (4.1) in each subinterval $\left[t_{j-1}, t_{j}\right]$, i.e.

$$
x_{i, j}(t)=\phi_{i, j}(t ; 1)=x_{i, j}\left(t^{*}\right)+\sum_{m=1}^{\infty} x_{i, j, m}(t) .
$$

\section{Numerical results}

In this work, we carefully propose the MHAM, a reliable modification of the HAM, that improves the convergence of the series solution. The method provides immediate and visible symbolic terms of analytic solutions, as well as numerical approximate solutions to both linear and nonlinear differential equations. Now, if we select the auxiliary linear operator $L=D^{\alpha}$, then the $\mathrm{m}^{\text {th }}$ order deformation equations (4.11) for the model of HIV infection of $\mathrm{CD} 4^{+} \mathrm{T}$ cells $(2.1)$ can be written in the form

$$
\begin{aligned}
\grave{T}_{j, m}(t) & =\chi_{m} \grave{T}_{j, m-1}(t)+h J^{\alpha}\left[\Re_{j, m}^{1}\left(\overrightarrow{\grave{T}}_{j, m-1}(t)\right)\right], \\
I_{j, m}(t) & =\chi_{m} I_{j, m-1}(t)+h J^{\alpha}\left[\Re_{j, m}^{1}\left(\vec{I}_{j, m-1}(t)\right)\right], \\
V_{j, m}(t) & =\chi_{m} V_{j, m-1}(t)+h J^{\alpha}\left[\Re_{j, m}^{1}\left(\vec{V}_{j, m-1}(t)\right)\right],
\end{aligned}
$$

where 


$$
\begin{gathered}
\Re_{j, m}^{1}\left(\vec{T}_{j, m-1}(t)\right)=D^{\alpha} \grave{T}_{j, m-1}(t)+\delta \grave{T}_{j, m-1}(t)-r \grave{T}_{j, m-1}(t) \\
+\frac{r}{\grave{T}_{m}}\left(\sum_{i=0}^{m-1} \grave{T}_{j, i}(t) \grave{T}_{j, m-i-1}(t)+\sum_{i=0}^{m-1} \grave{T}_{j, i}(t) I_{j, m-i-1}(t)\right) \\
+k \sum_{i=0}^{m-1} \grave{T}_{j, i}(t) V_{j, m-i-1}(t)-s\left(1-\chi_{m}\right), \\
\left.\Re_{j, m}^{2}\left(\vec{I}_{j, m-1}(t)\right)=D^{\alpha} I_{j, m-1}(t)-k \sum_{i=0}^{m-1} \grave{T}_{j, i}(t) V_{j, m-i-1}(t)\right)+\beta I_{j, m-1}(t), \\
\mathrm{j}=1,2, \ldots, \mathrm{n}, \\
\Re_{j, m}^{3}\left(\vec{V}_{j, m-1}(t)\right)=D^{\alpha} V_{j, m-1}(t)-N \beta I_{j, m-1}(t)+\gamma V_{j, m-1}(t) .
\end{gathered}
$$

So in this case we have to satisfy the initial condition at each of the subintervals. Accordingly, the initial values will be changed for each subinterval, i.e.

$$
\begin{gathered}
\grave{T}_{1}\left(t^{*}\right)=0.001, \grave{T}_{j}\left(t^{*}\right)=\grave{t}_{j}\left(t_{j-1}\right)=\grave{t}_{j-1}\left(t_{j-1}\right), \\
I_{1}\left(t^{*}\right)=0, I_{j}\left(t^{*}\right)=i_{j}\left(t_{j-1}\right)=i_{j-1}\left(t_{j-1}\right) \\
V_{1}\left(t^{*}\right)=0.001, V_{j}\left(t^{*}\right)=v_{j}\left(t_{j-1}\right)=v_{j-1}\left(t_{j-1}\right), j=2,3, \ldots, n .
\end{gathered}
$$

Where $t^{*}$ is the initial value for each subintervals. The solutions of system (2.1) in each subinterval $\left[t_{j-1}, t_{j}\right], j=1,2, \ldots, n$, has the form

$$
\begin{aligned}
& \grave{t}_{j}(t)=\sum_{m=0}^{\infty} \grave{T}_{j, m}\left(t-t_{j-1}\right), \\
& \mathrm{i}_{j}(t)=\sum_{m=0}^{\infty} I_{j, m}\left(t-t_{j-1}\right), j=1,2, \ldots, n, \\
& v_{j}(t)=\sum_{m=0}^{\infty} V_{j, m}\left(t-t_{j-1}\right),
\end{aligned}
$$

and the solution of system (2.1) for $[0, T]$ is given by

$$
\grave{T}(t)=\sum_{j=1}^{n} \chi_{v} \grave{t}_{j}(t),
$$




$$
\mathrm{I}(\mathrm{t})=\sum_{j=1}^{n} \chi_{v} i_{j}(t)
$$

$$
V(t)=\sum_{j=1}^{n} \chi_{v} v_{j}(t) .
$$

To demonstrate the effectiveness of the proposed algorithm as an approximate tool for solving the model of HIV infection of $\mathrm{CD}^{+} \mathrm{T}$ cells of fractional order (2.1) for larger t, we apply the proposed algorithm on the interval $[0,60]$. We choose to divide the interval $[0,60]$ into subintervals with time step $\Delta t=0.1$. and we get the HAM solution at each subinterval. Figs 1 and 2 show the phase portrait for the classical model for HIV infection of $\mathrm{CD}^{+} \mathrm{T}$ cells using the MHAM and the fourth-order RungeKutta method (RK4). From the graphical results in Figs. 1 and 2, it can be seen the results obtained using the MHAM match the results of the RK4 very well, which implies that the MHAM can predict the behavior of these variables accurately for the region under consideration. Figs. 3 and 4 show the phase portrait for the fractional-order model for HIV infection of $\mathrm{CD} 4^{+} \mathrm{T}$ cells using the MHAM. From the numerical results in Figs. 3 and 4 , it is clear that the approximate solutions depend continuously on the time-fractional derivative $\alpha$. The effective dimension $\sum$ of the system (5.1) is defined as the sum of orders $3 \alpha=\sum$. Also we can see that the chaos

exists in the fractional-order model for HIV infection of $\mathrm{CD} 4^{+} \mathrm{T}$ cells with order as low as 2.1 .

\section{Conclusions}

The analytical approximations to the solutions of the model of HIV infection of $\mathrm{CD}^{+} \mathrm{T}$ cells are reliable and confirm the power and ability of the MHAM as an easy device for computing the solution of nonlinear problems. In this paper, a fractional differential model of HIV is studied and its approximate solution is presented using a MHAM. The approximate solutions obtained by MHAM are highly accurate and valid for a long time. The reliability of the method and the reduction in the size of computational domain give this method a wider applicability. Finally, the recent appearance of nonlinear fractional differential equations as models in some fields such as models in science and engineering makes it is necessary to investigate the method of solutions for such equations and we hope that this work is a step in this direction. 


\section{References}

[1] A. K. Alomari, M. S. M. Noorani, R. Nazar, C. P. Li, Homotopy analysis method for solving fractional Lorenz system, Commun Nonliear Sci Numer Simult, 15 (7), pp. 1864-1872, (2010).

[2] J. Cang, Y. Tan, H. Xu, S. Liao, Series solutions of non-linear Riccati differential equations with fractional order, Chaos, Solitons \& Fractals, 40 (1), pp. 1-9, (2009).

[3] R.V. Culshaw, S. Ruan, A delay-differential equation model of HIV infection of $\mathrm{CD}^{+}{ }^{+}$T-cells, Mathematical Bioscience 165, pp. 27-39, (2000).

[4] Y. Ding, H. Ye, A fractional-order differential equation model of HIV infection of $\mathrm{CD} 4^{+}$T-cells, Mathematical and Computer Modelling, 50, pp. 386-392, (2009).

[5] V. S. Ertürk, Z. Odibat, S. Momani, An approximate solution of a fractional order differential equation model of human T-cell lymphotropic virus I (HTLV-I) infection of CD4 ${ }^{+}$T-cells, Computers \& Mathematics with Applications, 62, pp. 992-1002, (2011).

[6] C.P. Li, W.H. Deng, Remarks on fractional derivatives, Applied Mathematics and Computation 187, pp. 777-784, (2007).

[7] W. Lin, Global existence theory and chaos control of fractional differential equations, JMAA, 332, pp. 709-726, (2007).

[8] M. Merdan, homotopy perturbation method for solving a model for HIV infection of T-cells. Istanbul Ticaret Universitesi Fen Bilimleri Dergisi, 12, pp. 39-52, (2007).

[9] S. Miller, B. Ross, An Introduction to the Fractional Calculus and Fractional Differential Equations, John Wiley \& Sons, USA, (1993).

[10] K. B. Oldham, J. Spanier, The Fractional Calculus, Academic Press, New York, (1974).

[11] M. A. Nowak, R. M. May, Virus Dynamics, Oxford University Press, (2000). 
[12] A.S. Perelson, Modelling the interaction of the immune system with HIV, in: C. Castillo-Chavez (Ed.), Mathematical and Statistical Approaches to AIDS Epidemiology, Springer, Berlin, (1989).

[13] A. S. Perelson, D. E. Kirschner, R. De Boer, Dynamics of HIV infection of CD4 ${ }^{+}$T-cells, Math. Biosci, 114 (1), pp. 81-125, (1993).

[14] A.S. Perelson, P.W. Nelson, Mathematical analysis of HIV-1, dynamics in vivo, SIAM Rev. 41 (1), pp. 3-44, (1999).

[15] I. Podlubny, Fractional Differential Equations. Academic Press, New York, (1999).

[16] A. Rafiq, M. Rafiullah, Some multi-step iterative methods for solving nonlinear equations, Computers \& Mathematics with Applications, 58 (8), pp. 1589-1597, (2009).

[17] J. Sabatier, O .P. Agrawal, J. A. Tenreiro Machado, Advances in Fractional Calculus; Theoretical Developments and Applications in Physics and Engineering, Springer, (2007).

[18] M. Zurigat, S. Momani, Z. odibat, A. Alawneh, The homotopy analysis method for handling systems of fractional differential equations, Applied Mathematical Modelling, 34 (1), pp. 24-35, (2010).

[19] M. Zurigat, S. Momani, A. Alawneh, Analytical approximate solutions of systems of fractional algebraic-differential equations by homotopy analysis method, Computers and Mathematics with Applications, 59 (3), pp. 1227-1235, (2010).

\author{
Ali H. Handam \\ Department of Mathematics, \\ Al al-Bayt University \\ P. O. Box: 130095, \\ Al Mafraq, \\ Jordan \\ e-mail : ali.handam@windowslive.com
}


Asad A. Freihat

Applied Science Department,

Ajloun College Al-Balqa Applied University

Ajloun 26816,

Jordan

e-mail : asadfreihat@yahoo.com

and

M. Zurigat

Department of Mathematics,

Al al-Bayt University

P. O. Box: 130095,

Al Mafraq,

Jordan

e-mail : moh_zur@hotmail.com 


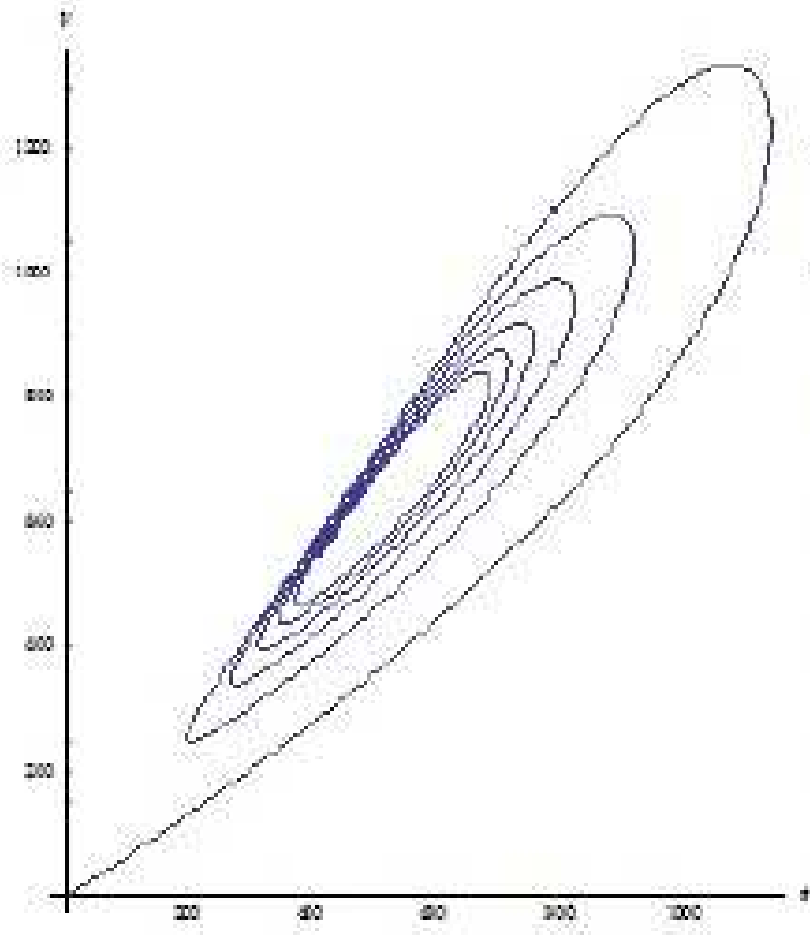

(a) МHAM

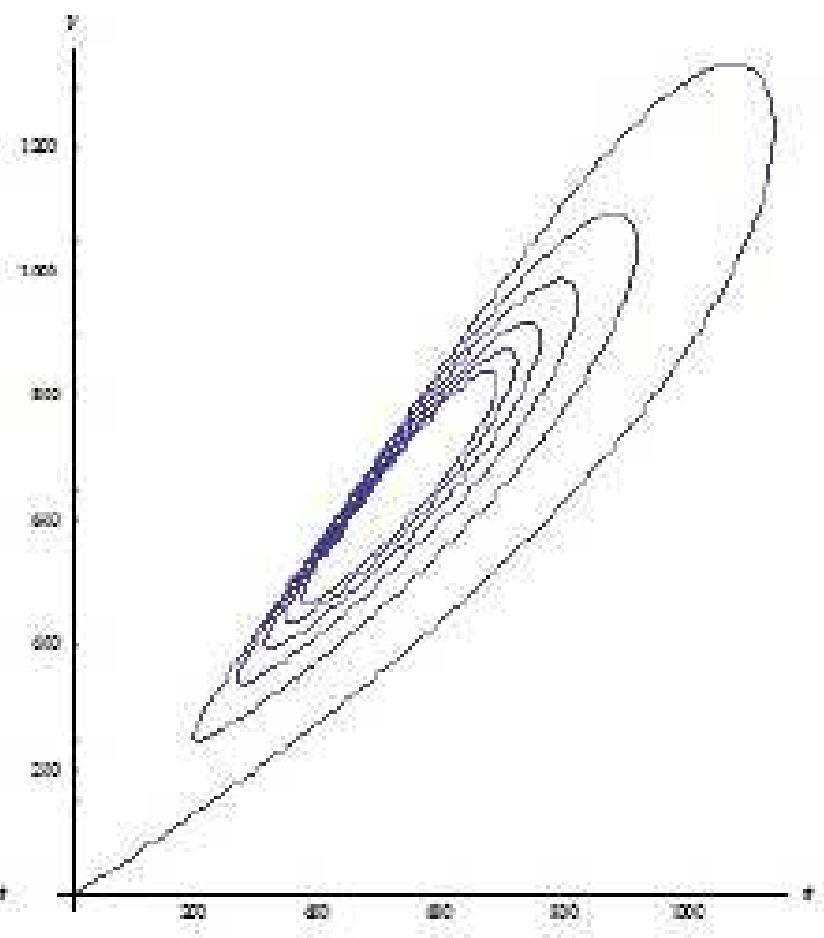

(b) RK4

Fig 1. Phave plot of $V(t)$ and $I(t)$, with $\alpha=1$.

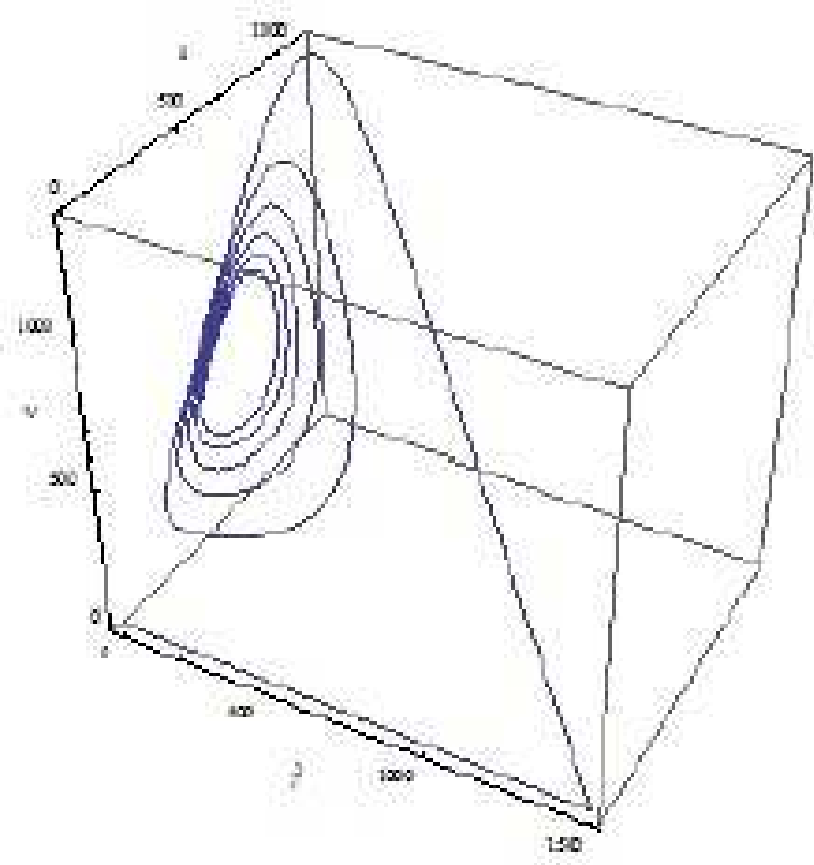

(a) MHAM

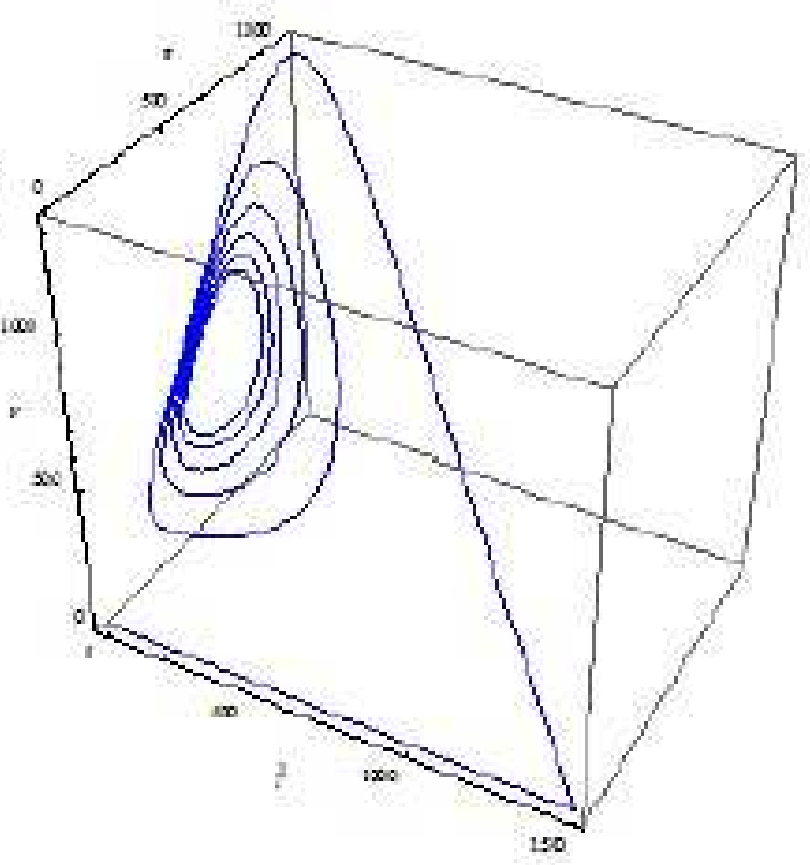

(5) RK4

Fig 2. Phase plot of $V(t), T(t)$ and $I(t)$, with $\alpha=1$. 


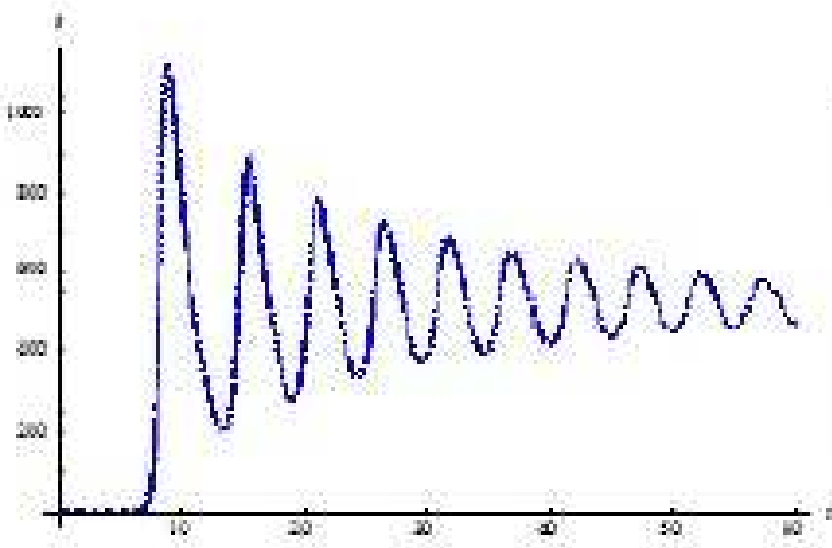

(a) $\alpha=0.86$

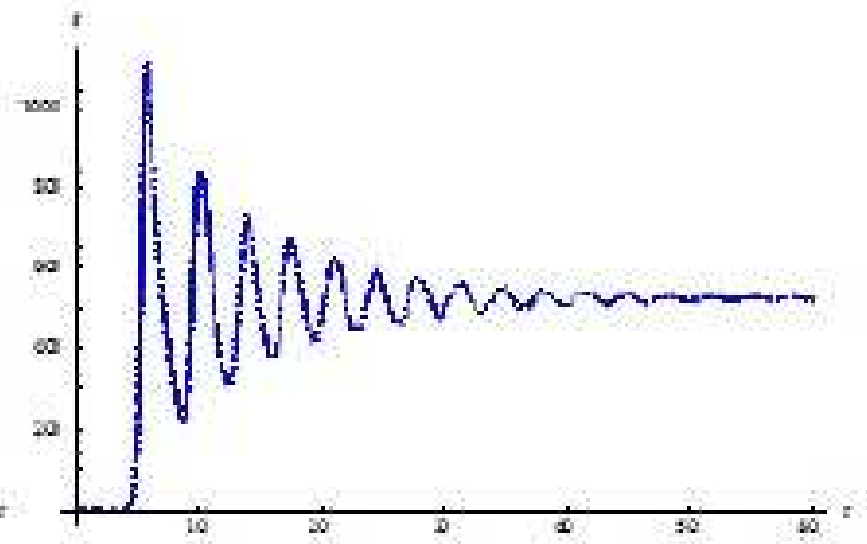

(b) $a=0.70$

Fig 3. $I(t)$ verous $t$.

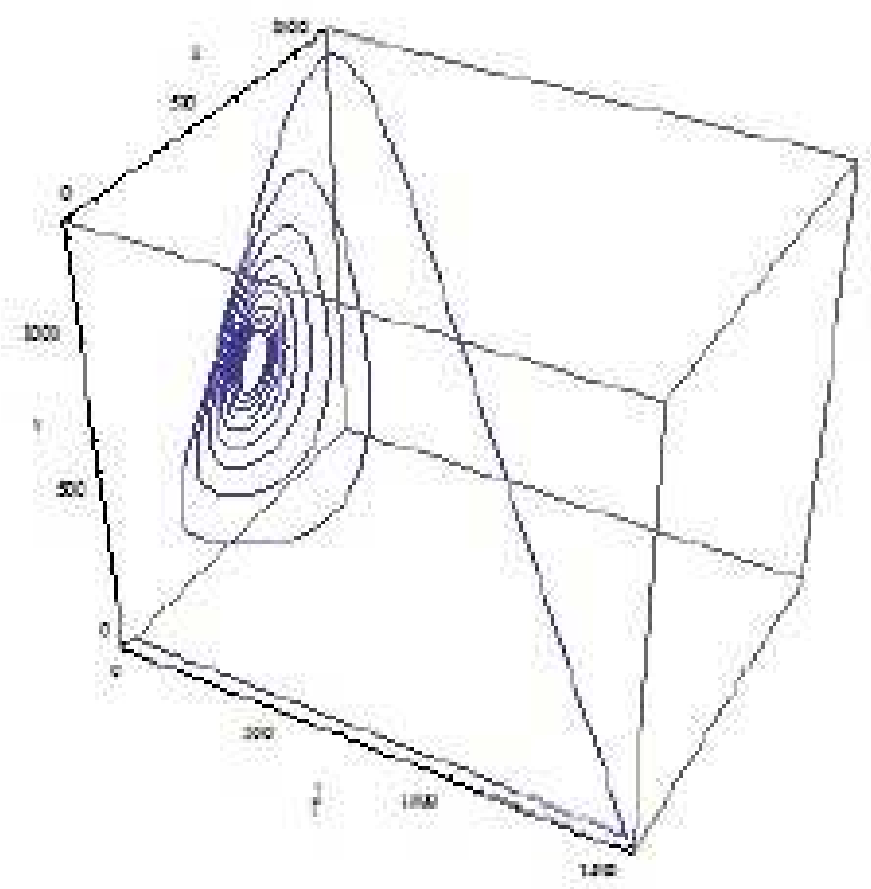

(a) $\alpha=0.85$

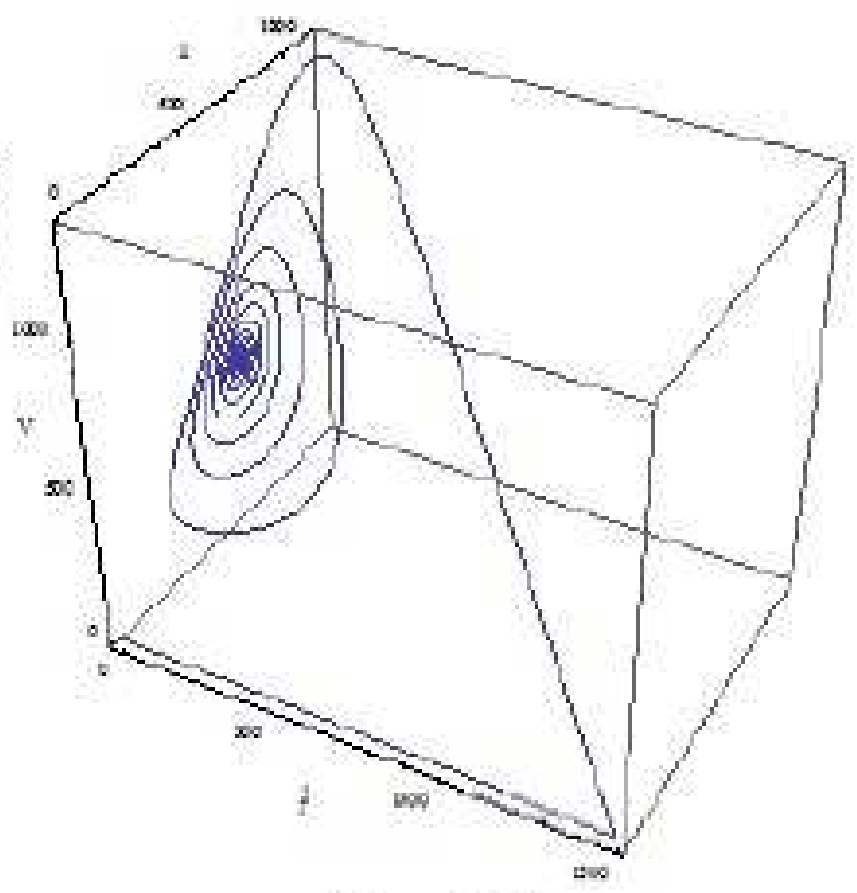

(b) $\alpha=0.70$

Fig 4. Phase plot of $V(t), T(t)$ and $I(t)$. 